\title{
Nonequilibrium many-body dynamics along a dissipative Hubbard chain: Symmetries and Quantum Monte Carlo simulations
}

\author{
L. Mühlbacher ${ }^{1}$ \\ ${ }^{1}$ School of Chemistry, The Sackler Faculty of Exact Science, Tel Aviv University, Tel Aviv 69978, Israel \\ J. Ankerhold ${ }^{2}$ \\ ${ }^{2}$ Physikalisches Institut, Albert-Ludwigs-Universität, D-79104 Freiburg, Germany
}

(Dated: January 30, 2019)

\begin{abstract}
The nonequilibrium dynamics of correlated charge transfer along a one-dimensional chain in presence of a phonon environment is investigated within a dissipative Hubbard model. For this generalization of the ubiquitous spin-boson model the crucial role of symmetries is analysed in detail and corresponding invariant subspaces are identified. It is shown that the time evolution typically occurs in each of the disjunct subspaces independently leading e.g. asymptotically to a non-Boltzmann equilibrium state. Based on these findings explicit results are obtained for two interacting electrons by means of a substantially improved real-time quantum Monte Carlo approach. In the incoherent regime an appropriate mapping of the many-body dynamics onto an isomorphic single particle motion allows for an approximate description of the numerical data in terms of rate equations. These results may lead to new control schemes of charge transport in tailored quantum systems as e.g. molecular chains or quantum dot arrays.

PACS numbers: 05.30.-d, 71.10.Fd, 05.10.-a
\end{abstract}

\section{INTRODUCTION}

In the last decade enormous progress has been achieved in fabricating designed quantum systems on the nanoscale. Typical examples include the assembling of quantum dot arrays to form artificial molecules [1, 2], the implementation of synthezised molecular structures in mesoscopic devices to develop molecular electronics [3, 4], and the tailoring of artificial atoms to obtain fully controllable quantum bits as the basis for quantum information processing 5]. A characteristic feature of such devices is that they consists of a relatively small number of relevant degrees of freedom, which are embedded in a much larger surrounding.

Of particular importance are electronic transport processes, which are then determined by an intimate interplay between electron-electron correlations and interactions with the environment. In this context, the simplest model, well-known as the so-called spin-boson model [6, 7], consists of a two-site chain where a single charge is transferred between the sites via tunneling hybridization. The charge dynamics can be coherent or incoherent depending on the interaction with the environmental degrees of freedom consisting e.g. of residual vibronic and phonon degrees of freedom or electromagnetic modes. In the past, the spin-boson model has been proven to capture essential properties of such electron transfer systems including various sorts of quantum phase transitions. Together with its extensions to multi-site systems it describes a broad field of phenomena comprising e.g. quantum Brownian motion 7], Kondo physics [8], Luttinger liquid behavior [7], atomic quantum dots [9], and charge transfer in photosynthesis [10]. However, a fundamental limitation of the spin-boson model is its restriction to single charge transfer, which becomes particularly severe, when the number of charges and their dwell time on a certain site can be controlled.

Recently, we studied a generalization to correlated charge transport based on a dissipative one-dimensional Hubbard model [11]. In its isolated form (no dissipation) the latter one has been extremely well analysed in various parameter domains and has served as a standard model to reveal the nature of many-body correlations 12]. While those studies have been performed mostly in the thermodynamic limit to determine equilibrium properties and quantum phase transitions, the situation in tailored quantum systems is different: here the dynamics far from equilibrium and in presence of a dissipative surrounding is to be investigated, where typically only few excess charges occupy a relatively small to moderate number of sites. One goal lies in the control of quantum properties in the transport, which inevitably happens to be influenced by energy exchange with and fluctuations from a phononic background.

The nonequilibrium dynamics of correlated charge transport in dissipative environments is an extremely challenging task. This is mainly due to the fact that on the one hand away from the limit of very weak interaction with the phonon bath perturbative approaches cannot be applied and on the other hand at lower temperatures phonon induced retardations become long-ranged. Hence, so far either completely coherent transfer (no or very weak dissipation) 13, 14] or completely sequential transfer in the limit of very strong Coulomb repulsion [15, 16], which effectively reduces to a single charge problem, has been considered.

In the last decade real-time path integral Monte Carlo techniques (PIMC) have been proven to provide an efficient and numerically exact tool to simulate the time evolution of dissipative tight-binding systems in ranges 
where perturbative schemes are prohibitive [7, 17]. The method has been substantially improved in the last years to tackle also larger systems (a larger number of sites) and many charges [18, 19]. These simulations are challenging in that the dimensionality of the Hilbert space to be sampled strongly increases with the number of particles, which in turn aggravates the so-called dynamical sign problem [20]. The latter one originates from the oscillatory character of the time evolution operators generating "signals" due to interferences. We note in passing that imaginary time path integral Monte Carlo approaches have already been successfully applied to extract thermodynamic properties of the Hubbard model 21, 22, 23. An alternative approach has been developed very recently by means of numerical renormalization group techniques (NRG) and its time dependent generalizations 24], which must be seen as being complementary to the PIMC scheme as the former ones are particularly adapted to the regime of very low temperatures, while the latter one shows its best efficiency in intermediate temperature domains.

The numerical results in Ref. 11] elucidated the crucial relevance of symmetries of the full Hamiltonian comprising system, bath, and interaction. Namely, it has been shown that the generic dipole-dipole coupling between many-electron system and bath leads to the existence of a decoherence free subspace (DFS), i.e. a subspace of the total Hilbert space which is invariant under the time evolution. Interestingly, similar kinds of DFSs have been analysed in the context of quantum information processing [5], however, in many-body systems their structure and impact on the transport dynamics is quite different. For instance, in contrast to the naive expectation for longer times the dissipative population dynamics of the many-body states approaches a steady state which is not of Boltzmann type and sensitively depends on the initial preparation. In this paper we give a more elaborate account of this work and provide a complete analysis of the symmetry properties of dissipative one-dimensional Hubbard chains. In fact, a DFS turns out to be just a special kind of an invariant subspace embedded into a whole class of invariant subspaces. This characterization provides a detailed understanding of the relaxation towards a non-Boltzmann type of equilibrium and possibly opens new control mechanisms for fermionic transport. It further serves in the regime of completely incoherent transfer as a starting point to approximate the dynamics of the reduced density matrix by simple master equations with transitions rates obtained from golden rule calculation, thus generalizing the rate concept known from single charge transfer 7]. Eventually, the existence of invariant subspaces may substantially improve the efficiency of the PIMC algorithm and particularly soothe the dynamical sign problem.

The paper is organized as follows. We start in Sec. II with the description of the dissipative Hubbard model. In the next two sections, Sec. III and Sec. IV] the symmetries of the model are analyzed in detail. This pro- vides in Sec. $\mathbb{V}$ insight into the dynamical and equilibrium populations, before we give a brief description of the PIMC method and its description in terms of a rate model in Sec. VI. Applications to specific realizations are discussed in Sec. VII At the end are short summary is given and some conclusions are drawn.

\section{DISSIPATIVE HUBBARD-MODEL}

The system is modeled by an open Hubbard chain with $N$ sites of spacing $q_{0}$

$$
\begin{aligned}
H_{S}= & \sum_{i=1, \sigma=\uparrow, \downarrow}^{N}\left(E_{i} d_{i, \sigma}^{\dagger} d_{i, \sigma}+\frac{U_{i}}{2} d_{i, \sigma}^{\dagger} d_{i, \sigma} d_{i,-\sigma}^{\dagger} d_{i,-\sigma}\right) \\
& +\sum_{i=1, \sigma=\uparrow, \downarrow}^{N-1} \Delta_{i}\left(d_{i, \sigma}^{\dagger} d_{i+1, \sigma}+\text { h.c. }\right)
\end{aligned}
$$

where $d_{i, \sigma}$ and $d_{i, \sigma}^{\dagger}$ are annihilation and creation operators for electrons, respectively, with spin $\sigma$ on the site $i$. $E_{i}$ are the bare energies of the levels, $U_{i}$ denotes the strengths of the Coulomb interaction on site $i$, and $\Delta_{i}$ are the tunneling matrix elements. The influence of the bosonic bath is given by a Caldeira-Leggett type of model [25], where the interaction with the bath

$$
H_{B}=\sum_{\alpha}\left(\frac{P_{\alpha}^{2}}{2 m_{\alpha}}+\frac{1}{2} m_{\alpha} \omega_{\alpha}^{2} X_{\alpha}^{2}\right)
$$

is accomplished via a standard dipole coupling [10]

$$
H_{I}=-q_{0} \mathcal{P} \sum_{\alpha} c_{\alpha} X_{\alpha}+q_{0}^{2} \mathcal{P}^{2} \sum_{\alpha} \frac{c_{\alpha}^{2}}{2 m_{\alpha} \omega_{\alpha}^{2}},
$$

where

$$
\mathcal{P}=\sum_{i=1, \sigma}^{N}[i-(N+1) / 2] d_{i, \sigma}^{\dagger} d_{i, \sigma}
$$

is the polarization operator of the Hubbard chain. This way, the above model can be seen as a generalization of the spin boson model [6, 7] of charge transfer to the case of many electrons and many sites.

In the sequel we deal exclusively with distinguishable charges and thus, in the case of fermions, concentrate on the two particle/opposite spin sector, see Ref. [11]. We note in passing though that all results presented in this paper are fully transferable to any two particles as long as they can be physically distinguished. Based on this analysis, the generalizations to more charges/particles may be tedious in detail, but straightforward in principle. The case of indistinguishable particles is related to the bosonic Hubbard model and will be discussed in a subsequent paper.

A suitable basis for the electronic degrees of freedom is now given in terms of the localized many-body states (LMBS)

$$
\mathcal{B}_{\text {loc }}=\left\{\left|s^{\downarrow}, s^{\uparrow}\right\rangle_{A}\right\}_{-S \leq s^{\downarrow}, s^{\uparrow} \leq S},
$$


where $\left|s^{\downarrow}, s^{\uparrow}\right\rangle_{A}$ denotes an antisymmetrized state with the $\sigma=\downarrow(\sigma=\uparrow)$ fermion being localized on site $i=$ $s^{\downarrow}+S+1\left(i=s^{\uparrow}+S+1\right)$, where $S=(d-1) / 2$, i.e.

$$
\left|s^{\downarrow}, s^{\uparrow}\right\rangle_{A}=\frac{1}{\sqrt{2}}\left(\left|1, \downarrow, s^{\downarrow}\right\rangle\left|2, \uparrow, s^{\uparrow}\right\rangle-\left|1, \uparrow, s^{\uparrow}\right\rangle\left|2, \downarrow, s^{\downarrow}\right\rangle\right),
$$

with, e.g., $\left|1, \downarrow, s^{\downarrow}\right\rangle$ denoting a state with the first particle being in the spin-down state, localized on site $i=s^{\downarrow}+$ $S+1$. Since only antisymmetrized states will be used throughout the rest of the paper, the subscript $A$ will be omitted in the following. The time evolution of the LMBS populations then follows from

$$
P_{s \downarrow, s^{\uparrow}}(t) \equiv\left\langle s^{\downarrow}, s^{\uparrow}|\rho(t)| s^{\downarrow}, s^{\uparrow}\right\rangle,
$$

where $\rho(t)=\operatorname{tr}_{B}\{W(t)\}$ denotes the reduced density matrix of the electronic system, obtained from the full density matrix $W(t)=\exp (-i H t / \hbar) W(0) \exp (i H t / \hbar)$ with $H=H_{S}+H_{B}+H_{I}$ after integrating out the environmental degrees of freedom (see also Sec. VIA).

As shown in 11, the reduced dynamics (7) is strongly determined by the symmetries of the underlying Hamiltonian of the total compound. In fact, the dipole type of system-bath coupling provides a symmetry which gives rise to a decoherence free subspace (DFS). The projector onto this subspace commutes with $H_{S}$ and $H_{I}$, i.e. with the polarization $\mathcal{P}$, meaning that it is not affected by the dissipative dynamics of the environment. Here we argue that such a DFS is actually just a special case of a more general property: The decomposition of the Hilbert space into invariant subspaces between which neither the free Hamiltonian (11) nor the bath can induce transitions. Accordingly, when populating initially only one of these subspaces, the dissipative system's dynamics remains completely restricted to it. This has profound consequences: (i) Only subspaces of the full Hilbert space must be sampled in the Monte Carlo simulations which substantially reduces the dynamical sign problem and (ii) for appropriate bath parameters the full quantum dynamics can be expressed into simple rate equations, which intrinsically obey the corresponding symmetries and lead to the correct equilibrium populations.

The analysis of the symmetry properties and its relation to invariant subspace is most conveniently done by exploiting that the $n$-particle dynamics on a onedimensional lattice can be mapped onto an effective single particle diffusion on a $n$ dimensional one. For instance, as shown in Ref. [26], for two distinguishable fermions on a one-dimensional lattice with $d$ sites a mapping exists onto one particle dynamics onto a twodimensional square lattice with $d^{2}$ sites, see Fig. 1 This mapping turns out to be a very convenient tool on the one hand to visualize the relation between manybody dynamics and dissipation and on the other it may serve as a starting point to apply perturbative methods as e.g. the non-interacting blip/cluster approximation (NIBA/NICA), which are known to be powerful means to capture the single particle motion. The rate equations

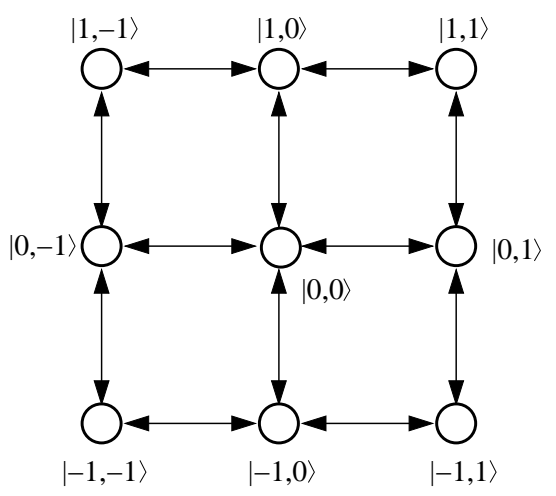

FIG. 1: 2d mapping of the LMBS (5) for $d=3$.

mentioned above are a direct consequence of this type of approximation.

\section{SPIN-PERMUTATION SYMMETRY}

Since the dissipative Hubbard Hamiltonian contains no terms depending on the fermions' spins, it is invariant with respect to any permutation of the latter. This leads to the most robust symmetry of the model, namely,

$$
[\mathcal{F}, H]=\left[\mathcal{F}, H_{S}\right]=\left[\mathcal{F}, H_{I}\right]=\left[\mathcal{F}, H_{B}\right]=0,
$$

where we introduced the spin-permutation operator

$$
\mathcal{F}\left|s^{\downarrow}, s^{\uparrow}\right\rangle=\left|s^{\uparrow}, s^{\downarrow}\right\rangle,
$$

which, for two particles, is equivalent to a simultaneous spin flip.

Note that $\mathcal{F}$ commutes not just with the total Hamiltonian $H$, but with $H_{0}$ and $H_{I}$ separately, such that we can easily find an electronic basis of simultaneous eigenstates of $\mathcal{F}$ and $\mathcal{P}$. For example

$$
\begin{aligned}
\mathcal{B}_{\text {flip }} \equiv & \mathcal{B}_{\text {flip }}^{+} \cup \mathcal{B}_{\text {flip }}^{-} ; \\
\mathcal{B}_{\text {flip }}^{+} \equiv & \left\{\left|s^{\downarrow}, s^{\uparrow}=s^{\downarrow}\right\rangle\right\}_{\mid s \downarrow} \mid \leq S \\
& \cup\left\{\frac{1}{\sqrt{2}}\left(\left|s^{\downarrow}, s^{\uparrow}\right\rangle+\left|s^{\uparrow}, s^{\downarrow}\right\rangle\right)\right\}_{s^{\downarrow}<s^{\uparrow}}, \\
\mathcal{B}_{\text {flip }}^{-} \equiv & \left\{\frac{1}{\sqrt{2}}\left(\left|s^{\downarrow}, s^{\uparrow}\right\rangle-\left|s^{\uparrow}, s^{\downarrow}\right\rangle\right)\right\}_{s^{\downarrow}<s^{\uparrow}},
\end{aligned}
$$

where $\mathcal{B}_{\text {flip }}^{ \pm}$only includes eigenstates of $\mathcal{F}$ with eigenvalue \pm 1 , respectively. Transitions between $\mathcal{B}_{\text {flip }}^{+}$and $\mathcal{B}_{\text {flip }}^{-}$are globally prohibited, independent of the system parameters $d, E_{i}, U_{i}, \Delta_{i}$, or any bath parameters. Therefore, the electronic Hilbert space $\mathcal{H}$ decomposes into two disjunctive invariant subspaces $\mathcal{G}^{ \pm} \equiv \operatorname{span}\left\{\mathcal{B}_{\text {flip }}^{ \pm}\right\}$, i.e.

$$
\mathcal{H}=\mathcal{G}^{+} \cup \mathcal{G}^{-}, \quad \mathcal{G}^{+} \cap \mathcal{G}^{-}=\varnothing, \quad H \mathcal{G}^{+} \subseteq \mathcal{G}^{+}, H \mathcal{G}^{-} \subseteq \mathcal{G}^{-},
$$


with distinct routes to thermal equilibrium. As a consequence, the LMBS populations (7) exhibit different relaxation time scales and, even at high temperatures, a nonBoltzmann equilibrium distribution. Furthermore, the latter explicitly depends on the initial preparation. For example, for an energetically completely degenerate system (i.e. $E_{i}=U_{i}=0$ ) the populations for the $d(d+1) / 2$ states in $\mathcal{B}_{\text {flip }}^{+}$equilibrate towards $2 P_{0}^{+} / d(d+1)$, while those for the $d(d-1) / 2$ states in $\mathcal{B}_{\text {flip }}^{+}$equilibrate towards $2 P_{0}^{-} / d(d-1)$, where $P_{0}^{ \pm}$denote the initial populations of the subspaces $\mathcal{G}^{ \pm}$, respectively $\left(P_{0}^{+}+P_{0}^{-}=1\right)$. Accordingly, even for sufficiently strong damping and/or high temperatures, the equilibrium populations of the localized states (5) do not coincide with a Boltzmann distribution.

For the special case $d=2$, it is straightforward to show that the basic spin-permutation symmetry fully explains the existence of a decoherence-free state as reported in Ref. 11] (namely in form of the only element of $\mathcal{B}_{\text {flip }}^{-}$). The robustness of this DFS with respect to changes in system and bath parameters directly follows from the robustness of the spin-permutation symmetry itself. For $d>2$ we postpone a more detailed discussion and first analyse in the next section additional symmetries related to particular parameter ranges for $E_{i}, U_{i}, \Delta_{i}$. We already note, though, that the spin-permutation symmetry can be destroyed e.g. by applying external magnetic fields, which in turn would also allow to control its influence on the many-body dynamics.

\section{PSEUDO-ANGULAR MOMENTUM BASIS AND INVARIANT SUBSPACES}

For the tight binding lattice with the localized basis (5), it is very suggestive to identify the discrete positions of the charges $s^{\downarrow}$ and $s^{\uparrow}$ with the discrete eigenvalues of the z-components $J_{z}^{\downarrow}$ and $J_{z}^{\uparrow}$, respectively, of two pseudo-angular momentum operators. Accordingly, the latter ones obey $\vec{J} \downarrow^{2}=\vec{J}^{\uparrow 2}=\hbar^{2} S(S+1)$ with $d=2 S+1$. For single charge transfer on two sites this leads to the famous spin-boson model where the polarization is then proportional to the Pauli matrix $\sigma_{z}$. Now, for two charges the polarization $\mathcal{P}$ is proportional to $J_{z}^{\downarrow}+J_{z}^{\uparrow}$. Hence, for the analysis of symmetry properties it is convenient to introduce an alternative basis of the fermionic Hilbert space following from a total pseudo-angular momentum $\vec{J}=\vec{J} \downarrow+\vec{J}^{\uparrow}$. The consequences of this formulation are studied in this section.

\section{A. Collective states}

Within the pseudo-angular momentum representation the localized states in $\mathcal{B}_{\text {loc }}(5)$ are simultaneous eigenstates of $\overrightarrow{J^{\downarrow}}, J_{z}^{\downarrow}, \vec{J}^{\uparrow 2}$, and $J_{z}^{\uparrow}$ due to

$$
\left|s^{\downarrow}, s^{\uparrow}\right\rangle \equiv\left|j^{\downarrow}=S, j^{\uparrow}=S ; m^{\downarrow}=s^{\downarrow}, m_{\uparrow}=s^{\uparrow}\right\rangle,
$$

while, as already mentioned, the polarisation operator (4) can be expressed as

$$
\mathcal{P}=\frac{J_{z}^{\uparrow}+J_{z}^{\downarrow}}{\hbar}=\frac{J_{z}}{\hbar} .
$$

Here $J_{z}$ denotes the $z$ component of $\vec{J}=\vec{J} \downarrow+\vec{J}^{\uparrow}$. An alternative electronic basis set is then given by the simultaneous eigenstates of these two operators, i.e.,

$$
\mathcal{B}_{\mathrm{J}}=\left\{\left|\psi_{m}^{(j)}\right\rangle_{0 \leq j \leq d-1,-j \leq m \leq j},\right.
$$

where $\overrightarrow{J^{2}}\left|\psi_{m}^{(j)}\right\rangle=\hbar^{2} j(j+1)\left|\psi_{m}^{(j)}\right\rangle$ and $J_{z}\left|\psi_{m}^{(j)}\right\rangle=$ $\hbar m\left|\psi_{m}^{(j)}\right\rangle$. Note that, like the localized states in $\mathcal{B}_{\text {loc }}$, the collective states in $\mathcal{B}_{\mathrm{J}}$ are also eigenstates of the polarization operator $\mathcal{P}(13)$, as well as of the spin-permutation operator $\mathcal{F}$ (9) $\left(\mathcal{F}\left|\psi_{m}^{(j)}\right\rangle=(-1)^{j+1}\left|\psi_{m}^{(j)}\right\rangle\right)$.

The transformation between the two basis sets is conveniently given in terms of the (real-valued) ClebschGordan coefficients [27] $\left\langle s^{\downarrow}, s^{\uparrow} \mid \psi_{m}^{(j)}\right\rangle \equiv\left\langle S, S, s^{\downarrow}, s^{\uparrow} \mid \psi_{m}^{(j)}\right\rangle$. Hence, defining

$$
C_{s^{\downarrow}, s^{\uparrow}}^{(j)} \equiv\left\langle s^{\downarrow}, s^{\uparrow} \mid \psi_{s^{\downarrow}+s^{\uparrow}}^{(j)}\right\rangle
$$

and exploiting $\left\langle s^{\downarrow}, s^{\uparrow} \mid \psi_{m}^{(j)}\right\rangle=0$ for $m \neq s^{\downarrow}+s^{\uparrow}$, we obtain

$$
\begin{aligned}
\left|\psi_{m}^{(j)}\right\rangle & =\sum_{s^{\downarrow}=\max \{-S, m-S\}}^{\min \{S, m+S\}} C_{s^{\downarrow}, m-s^{\downarrow}}^{(j)}\left|s^{\downarrow}, m-s^{\downarrow}\right\rangle, \\
\left|s^{\downarrow}, s^{\uparrow}\right\rangle & =\sum_{j=\left|s^{\downarrow}+s^{\uparrow}\right|}^{d-1} C_{s^{\downarrow}, s^{\uparrow}}^{(j)}\left|\psi_{s^{\downarrow}+s^{\uparrow}}^{(j)}\right\rangle .
\end{aligned}
$$

With the aid of Eq. 16] one can now easily express the LMBS populations (7) in terms of the corresponding ones in the pseudo-angular momentum basis,

$$
\hat{P}_{j, m}(t) \equiv\left\langle\psi_{m}^{(j)}|\rho(t)| \psi_{m}^{(j)}\right\rangle
$$

yielding

$$
\begin{aligned}
P_{s \downarrow, s \uparrow}(t) & =\sum_{\substack{j=\left|s^{\downarrow}+s^{\uparrow}\right| \\
s^{\downarrow}, s^{\uparrow}}}^{d-1} C_{j, s^{\downarrow}+s^{\uparrow}}^{(j)^{2}}(t) \\
+2 & \sum_{\substack{j, j^{\prime}=\left|s^{\downarrow}+s^{\uparrow}\right| \\
j^{\prime}>j}}^{d-1} C_{s^{\downarrow}, s^{\uparrow}}^{(j)} C_{s^{\downarrow}, s^{\uparrow}}^{\left(j^{\prime}\right)} \operatorname{Re}\left\{\left\langle\psi_{s^{\downarrow}+s^{\uparrow}}^{\left(j^{\prime}\right)}|\rho(t)| \psi_{s^{\downarrow}+s^{\uparrow}}^{(j)}\right\rangle\right\}
\end{aligned}
$$

Equation (18) also governs the transformation of the initial populations between the two basis sets. Particularly simple expressions are gained for an initially factorizing system-bath state where, as in many experiments, the system is prepared to reside on some localized state $\left|s_{0}^{\downarrow}, s_{0}^{\uparrow}\right\rangle$. The corresponding initial populations $\hat{P}_{j, m}(0)$ are then obtained from

$$
\hat{P}_{j, m}(0)=\left\{\begin{array}{cl}
C_{s_{0}^{\downarrow}, s_{0}^{\uparrow}}^{(j)^{2}} & \text { if } m=s_{0}^{\uparrow}+s_{0}^{\downarrow}, j \geq\left|s_{0}^{\uparrow}+s_{0}^{\downarrow}\right|, \\
0 & \text { else. }
\end{array}\right.
$$




\section{B. Invariant subspaces}

The formulation based on the total pseudo-angular momentum now allows to identify a further symmetry of the dissipative Hubbard model. For this purpose we first consider the case, where the full Hamiltonian $H$ commutes with the total pseudo-angular momentum $\vec{J}^{2}$, thus leading to invariant subspaces similar to the symmetry noted in Sec. III Classically speaking, $H$ preserves the angle between the individual pseudo-angular momenta $J^{\downarrow}$ and $J^{\uparrow}$.

Accordingly, we look for a representation of the electronic Hamiltonian $H_{S}$ in terms of the components $J_{x}$, $J_{y}, J_{z}$. Turning first to the nearest-neighbor hopping term $H_{S}^{\Delta} \equiv \sum_{i, \sigma} \Delta_{i}\left(d_{i, \sigma}^{\dagger} d_{i+1, \sigma}+h . c.\right)$, it is straightforward to show that with respect to $\mathcal{B}_{\text {loc }}$, it exhibits the same structure as $J_{x}$, namely,

$$
\left\langle\hat{s}^{\downarrow}, \hat{s}^{\uparrow}\left|H_{S}^{\Delta}\right| s^{\downarrow}, s^{\uparrow}\right\rangle=0 \quad \Leftrightarrow \quad\left\langle\hat{s}^{\downarrow}, \hat{s}^{\uparrow}\left|J_{x}\right| s^{\downarrow}, s^{\uparrow}\right\rangle=0,
$$

provided all couplings $\Delta_{i}$ are non-zero.

Since the non-vanishing matrix elements of $J_{x}$ are given by

$$
\left\langle s^{\downarrow} \pm 1, s^{\uparrow}\left|J_{x}\right| s^{\downarrow}, s^{\uparrow}\right\rangle=\frac{\hbar}{2} \sqrt{S(S+1)-s^{\downarrow}\left(s^{\downarrow} \pm 1\right)}
$$

(and $\left\langle s^{\downarrow}, s^{\uparrow} \pm 1\left|J_{x}\right| s^{\downarrow}, s^{\uparrow}\right\rangle$ accordingly), one finds that $H_{S}^{\Delta} \propto J_{x}$ is always true for $d=2$. For $d>2$ this holds only if the tunnel couplings (hopping amplitudes) are chosen properly, e.g. for $d=3$ one must have $\Delta_{1}=\Delta_{2}$, i.e. isotropic coupling, and $d \geq 4$ requires quite specific values for the coupling strengths. We will discuss this point in more detail below.

Next, we turn to the on-site terms in $H_{S}$ comprising the on-site energies and the Coulomb interactions. Since the latter one effectively gives a contribution to the former ones, we find that if $E_{i}$ and $U_{i}, i=1, \ldots N$, are distributed up to an overall factor according to the matrix elements of $J_{z}$, i.e.,

$$
\left\langle\hat{s}^{\downarrow}, \hat{s}^{\uparrow}\left|J_{z}\right| s^{\downarrow}, s^{\uparrow}\right\rangle=\left(s^{\downarrow}+s^{\uparrow}\right) \delta_{\hat{s}^{\downarrow}=s^{\downarrow}} \delta_{\hat{s}^{\uparrow}=s^{\uparrow}},
$$

then the electronic Hamiltonoperator can be cast into the form

$$
H_{S}=E J_{z}+\hat{\Delta} J_{x}
$$

with appropriate constants $E$ and $\hat{\Delta}$. It then follows immediately that

$$
\left[H, \vec{J}^{2}\right]=\left[H_{S}, \vec{J}^{2}\right]=\left[H_{I}, \vec{J}^{2}\right]=\left[H_{B}, \vec{J}^{2}\right]=0 .
$$

The total pseudo-angular momentum is thus a conserved quantity with respect to the full dissipative system. Equivalently, for a dissipative Hubbard model with $H_{S}$ of the form (23) both the free system as well as the bath can induce only transitions between states with the same total pseudo-angular momentum. Of course,
Eq. (23) is a sufficient but not necessary condition for Eq. (24) to hold. Obviously, any $H_{S}$ and $H_{I}$ where the system part can be written in terms of $J_{x}, J_{y}$, and $J_{z}$ (and powers of these operators) fulfill Eq. (24); in particular, the coupling between system and bath may also be nonlinear in the system operator. Indeed, below we will present an example where Eq. (23) does not hold, while the latter relation still applies.

With respect to the reduced electronic dynamics, Eq. (24) leads to consequences similar to those described in Sec. III First, the electronic subspace $\mathcal{H}$ of the full Hilbert space can be further decomposed into disjunctive invariant subspaces,

$$
\mathcal{H}=\cup_{j=0}^{d-1} \mathcal{G}_{j},
$$

where $\mathcal{G}_{j}$ denotes the subspace spanned by all eigenstates of $\vec{J}^{2}$ with a fixed total pseudo-spin $j$,

$$
\mathcal{G}_{j}=\operatorname{span}\left\{\left|\psi_{-j}^{(j)}\right\rangle, \ldots,\left|\psi_{j}^{(j)}\right\rangle\right\}
$$

with the properties

$$
\mathcal{G}_{k} \cap \mathcal{G}_{j}=\varnothing \quad \text { for all } k \neq j, \quad H \mathcal{G}_{j} \subseteq \mathcal{G}_{j} .
$$

To put it differently, with the above decomposition we have identified the irreducible representations of the sum of two angular momentum operators each with angular momentum $S=(d-1) / 2$. Unlike the spin-permutation symmetry, now the number of invariant subspaces equals $d$, thus explicitly depending on the length of the tight binding lattice. Furthermore, the structure of these subspaces turns out to resemble linear chains since due to

$$
\begin{aligned}
& \left\langle\psi_{m^{\prime}}^{(j)}\left|H_{S}\right| \psi_{m}^{(j)}\right\rangle=E \delta_{m^{\prime}=m} \\
& +\frac{\hat{\Delta}}{2}\left(\sqrt{j(j+1)-m(m+1)} \delta_{m^{\prime}=m+1}\right. \\
& \left.\quad+\sqrt{j(j+1)-m(m-1)} \delta_{m^{\prime}=m-1}\right) .
\end{aligned}
$$

Transitions thus are only possible between nearest neighbors with respect to $m$ between states $\left|\psi_{m}^{(j)}\right\rangle$ with fixed $j$.

To summarize the main outcome of this analysis, we have shown that an electronic Hamiltonian obeying (23) leads to a Hilbert space basis which can be decomposed into one singlet part, one triplet part, one quintet part, $\ldots$, up to a $2(d-1)+1$ part, each of which has a linear structure and between which transitions induced by the Hamiltonian are not possible whatsoever (see Fig. 2). The subspaces solely interact via the coupling to a common bath. The singlet space is exceptional in that it is an eigenstate of $H_{I}$ with vanishing eigenvalue for the polarization operator, thus being protected by any dynamical time evolution of the dissipative system, i.e. a DFS.

\section{POPULATIONS OF THE LOCALIZED MANY-BODY STATES}

For transport processes the populations of the LMBS are of particular relevance. Here, we show how the exis- 


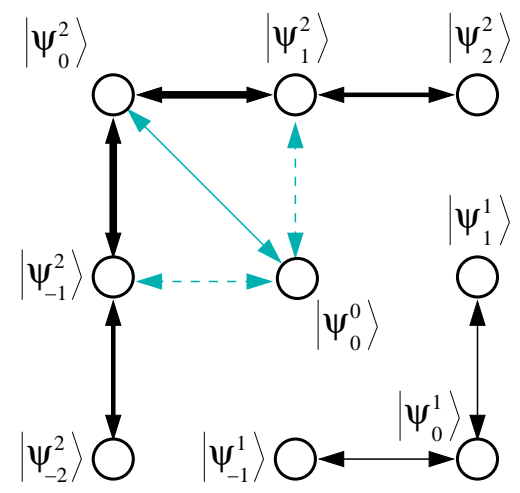

FIG. 2: 2d mapping for $d=3$ and the angular-momentum states (14). Black lines indicate the couplings for $\epsilon_{i}=U=0$; the thickness of the lines is proportional to the square of the coupling strengths. Gray solid lines indicate additional couplings for $U \neq 0, \epsilon_{i} \neq 0$; gray dashed lines indicate additional couplings for anisotrope couplings $\Delta_{1} \neq \Delta_{2}$.

tence of the ideal symmetry (24) may simplify their calculation considerably. This gives also direct access to the stationary equilibrium populations which are approached for sufficiently long times.

\section{A. Symmetries and population dynamics}

Due to the disjunct structure of the full Hilbert space, see Eq. (27), not only the total population is conserved, but also the individual ones within each invariant subspace. In particular, for a factorizing initial preparation $W(0)=\rho_{0} \exp \left(-\beta H_{B}\right)$, where $\rho_{0}=\left|\varphi_{0}\right\rangle\left\langle\left|\varphi_{0}\right|\right.$ presents a projector on some pure fermionic state $\left|\varphi_{0}\right\rangle$, subspaces with no overlap with $\left|\varphi_{0}\right\rangle$ do not participate in the dynamics at all. This leads to a reduction of the dimensionality of the relevant Hilbert space and thus of the complexity of the system to be treated numerically. If the initial electronic population is even restricted to a single subspace, i.e. $\left|\varphi_{0}\right\rangle \in \mathcal{G}_{j}$, the corresponding dynamics is identical to that of a single particle moving on a linear chain with just $2 j+1$ sites. For $\left|\varphi_{0}\right\rangle \in \mathcal{B}_{\text {loc }}$, this scenario only applies to the states $|-S,-S\rangle$ (i.e. $\left|\psi_{-(d-1)}^{(d-1)}\right\rangle$ ) and $|S, S\rangle$ (i.e. $\left|\psi_{d-1}^{(d-1)}\right\rangle$ ) with $d=j+1$. We note in passing that this property also applies to the situation $\left|\varphi_{0}\right\rangle \in \mathcal{G}_{j} \cup \mathcal{G}_{0}$ since $\mathcal{G}_{0}$ does not exhibit any dynamical evolution at all. For other initial preparations within $\mathcal{B}_{\text {loc }}$, however, direct phonon induced couplings between the participating subspaces mediated by the common environment [26] have to be taken into account, which do influence the dynamics but conserve the population in each subspace. For initial states which have overlaps with all invariant subspaces (i.e. $|-S, S\rangle$ or $|S,-S\rangle$ ), the dynamics only takes places in a $\left(d^{2}-1\right)$ dimensional subspace since again $\mathcal{G}_{0}$ does not participate.
Due to the invariance of the subspaces $\mathcal{G}_{j}$ with respect to $H$, the cross terms $\left\langle\psi_{s \downarrow+s^{\uparrow}}^{\left(j^{\prime}\right)}|\rho(t)| \psi_{s^{\downarrow}+s^{\uparrow}}^{(j)}\right\rangle$ in Eq. (18) are only non-vanishing if the initial state $\left|\varphi_{0}\right\rangle$ has an overlap with both $\mathcal{G}_{j}$ and $\mathcal{G}_{j^{\prime}}$. For most preparations in a LMBS, this significantly simplifies Eq. (18). This holds especially for preparations where both fermions enter at the same end of the chain (i.e. $|-S,-S\rangle$ and $|S, S\rangle$, both $\in \mathcal{G}_{d-1}$ ), when all localized populations can be expressed in terms of the pseudo-angular momentum populations alone. For all other localized preparations, however, the cross terms play a crucial role in the short-to-intermediate time domain. On the other hand, using Eq. (18) to obtain information about these cross terms also eludicates the dynamical evolution of coherences in the system.

\section{B. Equilibrium populations}

For long times a dissipative system reaches a stationary state, typically a thermal equilibrium where all states are occupied according to a Boltzmann distribution. In the case studied here, namely, a dissipative Hubbard model with the symmetry relations (24), the same applies not to the total Hilbert space, but rather its disjunct subspaces. In particular, for a state $\left|\psi_{m}^{(j)}\right\rangle \in \mathcal{G}_{j}$ one obtains

$$
\hat{P}_{j, m}^{\infty} \equiv \lim _{t \rightarrow \infty} \hat{P}_{j, m}(t)=\hat{P}_{0}^{(j)} \frac{e^{-\hbar \beta \hat{\epsilon}_{m}^{(j)}}}{\sum_{m^{\prime}=-j}^{j} e^{-\hbar \beta \hat{\epsilon}_{m^{\prime}}^{(j)}}},
$$

where the initial population in $\mathcal{G}_{j}$ is $\hat{P}_{0}^{(j)} \equiv$ $\sum_{m=-j}^{j} \hat{P}_{j, m}(0)$, and $\hbar \hat{\epsilon}_{m}^{(j)} \equiv\left\langle\psi_{m}^{(j)}\left|H_{S}\right| \psi_{m}^{(j)}\right\rangle$. Accordingly, the final occupations of the states $\left|\psi_{m}^{(j)}\right\rangle$ do not obey a Boltzmann distribution, i.e.,

$$
\frac{\hat{P}_{j, m}^{\infty}}{\hat{P}_{j^{\prime}, m^{\prime}}^{\infty}} \neq e^{-\hbar \beta\left(\hat{\epsilon}_{m}^{(j)}-\hat{\epsilon}_{m^{\prime}}^{\left(j^{\prime}\right)}\right)} .
$$

The same is true for the states in $\mathcal{B}_{\text {loc }}$. Since in thermal equilibrium all cross terms in Eq. (18) vanish $\left(P_{s^{\uparrow}, s^{\downarrow}}^{\infty}=\right.$ $\left.P_{s^{\downarrow}, s^{\uparrow}}^{\infty}\right)$, we have

$$
P_{s^{\downarrow}, s^{\uparrow}}^{\infty}=\sum_{j=\left|s^{\downarrow}+s^{\uparrow}\right|}^{d-1} C_{s^{\downarrow}, s^{\uparrow}}^{(j)^{2}} \frac{\hat{P}_{0}^{(j)}}{Z_{j}} e^{-\hbar \beta \hat{\epsilon}_{s \downarrow+s}^{(j)} \uparrow}
$$

with $Z_{j}=\sum_{m^{\prime}=-j}^{j} e^{-\hbar \beta \hat{\epsilon}_{m^{\prime}}^{(j)}}$. Apparently, the equilibrium populations strongly depend on the initial preparation, i.e. on $\hat{P}_{0}^{(j)}$.

\section{QUANTUM MONTE-CARLO SIMULATIONS}

So far we have focused on a convenient representation of the states within the fermionic subspace of the total Hilbert space. To obtain the populations Eqs. (7) 
and (17) explicitly, however, one must find an adequat treatment of the environmental degrees of freedom as well. The path integral approach has been proven to allow for an exact elimination of the latter and to provide in combination with Monte Carlo techniques a numerically exact evaluation.

\section{A. Path integral representation}

The expectation value of a system observable $A$

$$
\langle A(t)\rangle=\operatorname{Tr}_{S}\{\rho(t) A\}
$$

requires the knowledge of the reduced density operator

$$
\rho(t)=\operatorname{Tr}_{\mathrm{B}}\left\{\mathrm{e}^{-i H t / \hbar} W(0) \mathrm{e}^{i H t / \hbar}\right\} .
$$

Here, $H$ denotes the full Hamiltonian (1)-(3), $W(0)$ is an initial density, and the trace is performed over the bath degrees of freedom only. The details of the path integral representation for $\rho(t)$ have been given elsewhere $6,7,17,18$. Here, the standard expression known e.g. for the spin-boson model has to be extended to capture the dynamics of two distinguishable fermions. Accordingly, the reduced density operator is expressed as a double path integral along a Keldysh contour with forward $s^{\sigma}$ and backward $\tilde{s}^{\sigma}$ paths corresponding to the basis set $\mathcal{B}_{\text {loc }}$. The impact of the dissipative environment appears as an influence functional introducing arbitrarily long-ranged interactions in time along and between the paths. It is convenient to switch to the sum and difference coordinates $\eta^{\sigma}=s^{\sigma}+\tilde{s}^{\sigma}$ and $\xi^{\sigma}=s^{\sigma}-\tilde{s}^{\sigma}$, respectively, so that one arrives for the expectation value at the exact expression

$$
\langle A(t)\rangle=\oint \mathcal{D} \vec{\eta} \oint \mathcal{D} \vec{\xi} a[\vec{\eta}, \vec{\xi}] \mathcal{K}[\vec{\eta}, \vec{\xi}] \exp \{-\Phi[\vec{\eta}, \vec{\xi}]\}
$$

where $a[\vec{\eta}, \vec{\xi}]$ is the measurement functional corresponding to $A$ in terms of the combined system paths $\vec{\eta}(t)=$ $\left(\eta^{\uparrow}(t), \eta^{\downarrow}(t)\right)$ and $\vec{\xi}(t)=\left(\xi^{\uparrow}(t), \xi^{\downarrow}(t)\right)$. Furthermore, $\mathcal{K}$ is the bare action factor in absence of a reservoir, and the influence functional reads

$$
\begin{aligned}
& \Phi[\vec{\eta}, \vec{\xi}]= \int_{0}^{t} d s \int_{0}^{s} d u\left\{[\vec{\xi}(s) \cdot \vec{e}] L^{\prime}(s-u)[\vec{\xi}(u) \cdot \vec{e}]\right. \\
&\left.+i[\vec{\xi}(s) \cdot \vec{e}] L^{\prime \prime}(s-u)[\vec{\eta}(u) \cdot \vec{e}]\right\} \\
&+i \frac{\mu}{2} \int_{0}^{t} d s[\vec{\xi}(s) \cdot \vec{e}][\vec{\eta}(s) \cdot \vec{e}]
\end{aligned}
$$

with $\vec{e}=(1,1)$. The kernel $L(t)=L^{\prime}(t)+i L^{\prime \prime}(t)$ is related to the force-force auto-correlation function of the bath and is completely determined by the spectral density of its modes (A7). Further, $\mu=\lim _{\hbar \beta \rightarrow 0} \hbar \beta L(0)$. Note that even in absence of Coulomb interaction the two charges are correlated due to the coupling to the common heat bath.
An analytical treatment of the expression (34) is in general not feasible, mainly due to the retardations in the influence functional which grow with decreasing temperature, roughly as $\hbar \beta$. In this situation PIMC methods have been shown to be very powerful and numerically exact means to explore the non-perturbative range. Appendix A explains in some detail our approach which is straightforwardly obtained from the formally very similar case of a single dissipative particle [6, 7, 17, 18] and which can be easily extended to the cases of more than two or indistinguishable particles [26].

A striking difference to the single-particle case, however, is the existence of symmetries as described above and the consequent decomposition of the full system's Hilbert state into invariant subspaces. To exploit these symmetries for our numerical studies, we express Eq. (34) in terms of system paths $J(t)$ and $M_{J}(t)$, referring to the states $\left|\psi_{M}^{(J)}\right\rangle$ (14) rather than in terms of $s^{\downarrow}(t)$ and $s^{\uparrow}(t)$. It turns out that the environmental influence can again be summarized in an influence functional which is obtained from Eq. 35 by simply replacing $\vec{\eta}(s) \cdot \vec{e}$ and $\vec{\xi}(s) \cdot \vec{e}$ with $\eta_{M} \equiv M_{J}(t)+\tilde{M}_{J}(t)$ and $\xi_{M} \equiv M_{J}(t)-\tilde{M}_{J}(t)$, respectively, where $M_{J}(t)$ and $\tilde{M}_{J}(t)$ again denote paths on the forward and backward part of the Keldysh contour, respectively. The corresponding influence functional then reads

$$
\begin{aligned}
\Phi\left[\eta_{M}, \xi_{M}\right]= & \int_{0}^{t} d s \int_{0}^{s} d u\left[\xi_{M}(s) L^{\prime}(s-u) \xi_{M}(u)\right. \\
& \left.+i \xi_{M}(s) L^{\prime \prime}(s-u) \eta_{M}(u)\right] \\
& +i \frac{\mu}{2} \int_{0}^{t} d s \xi_{M}(s) \eta_{M}(s) .
\end{aligned}
$$

Note that for fixed $J$ this is just the influence functional of a single dissipative particle residing on $2 J+1$ discrete states.

Now, if Eq. (24) holds, $J(t)$ is conserved upon propagation with $\exp ( \pm i t H / \hbar)$ (see Eq. A1 ). A change in $J(t)$ can thus only be mediated by the initial preparation $\operatorname{Tr}\{W(0)\}$ and the measurement $a\left[\eta_{M}, \xi_{M}\right]$ at time $t$; otherwise $J(t)$ remains constant. In terms of paths of $J$ and $M$, Eq. (34) therefore eventually becomes

$$
\begin{aligned}
\langle A(t)\rangle= & \sum_{J_{1}, J_{2}=0}^{d-1} A_{J_{1}, J_{2}}(t), \\
A_{J_{1}, J_{2}}(t)= & \oint \mathcal{D} \eta_{M} \oint \mathcal{D} \xi_{M} a_{J_{1}, J_{2}}\left[\eta_{M}, \xi_{M}\right] \\
& \times \mathcal{K}_{J_{1}, J_{2}}\left[\eta_{M}, \xi_{M}\right] \exp \left\{-\Phi\left[\eta_{M}, \xi_{M}\right]\right\}
\end{aligned}
$$

where $J_{1}$ and $J_{2}$ denote the values of the piecewise constant $J$ paths along the forward and backward parts of the Keldysh contour, respectively, and $a_{J_{1}, J_{2}}\left[\eta_{M}, \xi_{M}\right]$ is the measurement functional of the operator $\mathbb{P}_{J_{1}} A \mathbb{P}_{J_{2}}$, with the projector $\mathbb{P}_{j}$ onto the subspace $\mathcal{G}_{j}$.

Further simplification can be achieved by exploiting that $A_{J_{1}, J_{2}}(t) \equiv 0$ if any of the subspaces $\operatorname{Tr}_{\mathrm{B}}\{W(0)\} \mathcal{G}_{J_{1}}$, $\operatorname{Tr}_{\mathrm{B}}\{W(0)\} \mathcal{G}_{J_{2}}$ or $A \mathcal{G}_{J_{1}}, A \mathcal{G}_{J_{2}}$ are empty. For example, 
if the system is initially prepared in the localized state $|-S,-S\rangle$, one simply obtains

$$
\langle A(t)\rangle=A_{d-1, d-1}(t) .
$$

Accordingly, in cases where the initial preparation does not impose transitions between subspaces with different $J$, the dissipative two-particle system can be decomposed into $d$ independent dissipative single-particle systems. For PIMC simulations this presents a major progress since each of the systems representing the $A_{J_{1}, J_{2}}(t)$ contributions exhibits a significantly lower dimensionality than the full $d^{2}$ dimensional two-particle system. Hence, by evaluating Eq. (37) rather than Eq. (34), the dynamical sign problem 20], which stems from interferences between different quantum paths and grows exponentially with the size of the system, can be greatly reduced. In the quantum regime this allows for substantial computational savings, despite the fact that the evaluation of Eq. (37) requires $d(d-1) / 2$ independent PIMC simulations (note $A_{J_{2}, J_{1}}(t)=A_{J_{1}, J_{2}}^{*}(t)$ ).

In case that Eq. (24) does not apply, an expression similar to Eq. (37) can still be obtained exploiting the spin-permutation symmetry. Therefore, the arguments presented above concerning the computational costs of PIMC simulations still apply, albeit to a lower degree.

For further details of the approach and particularly of the strategy to soothe the dynamical sign problem we refer to the literature [17, 18, 19]. The basic ingredients are this: (i) One exploits the fact that the influence functional depends only linearly on the quasi-classical paths $\vec{\eta}(t)$, so that in Eq. (34) the corresponding summations can be expressed as a series of simple matrix multiplications and therefore be carried out explicitly; (ii) for the sampling procedure one chooses an MC weight with no long-time retardations. Since they are fully taken into account in the final accumulation process evaluating the exact expression (34), the numerical exactness of the MC scheme is not impaired. This way, one achieves a strong decoupling of quasi-classical $(\vec{\eta})$ and quantum $(\vec{\xi})$ coordinates, which allows to store products of short time propagators independent of the MC-sampling. Eventually a speed-up with respect to the original method [17] by a factor of about 100 is gained. Of course, this strategy can be applied for evaluating both Eq. (34) as well as Eq. (37).

\section{B. Rate model}

For one-dimensional tight-binding systems a description of the single particle population dynamics in terms of simple master equations with transition rates gained from golden rule calculations is known to be quite accurate for sufficiently strong dissipation and/or high temperatures (incoherent dynamics) [7, 19]. Then, the exact dynamics approximately obeys

$$
\frac{d \vec{P}(t)}{d t}=\mathbf{R} \vec{P}(t)
$$

where $\vec{P}$ collects the single particle populations and the matrix $\mathbf{R}$ contains the transition rates between adjacent sites. These rates obey detailed balance reflecting the existence of a thermodynamic equilibrium approached for long times, where the populations are Boltzmann distributed such that $\mathbf{R} \vec{P}(t \rightarrow \infty)=0$. However, as we have shown in the previous sections, for the many-body time evolution this is no longer true, and formulating Eq. (39) with $\vec{P}(t)=\left(P_{-S,-S}(t), P_{-S,-S+1}(t), \ldots\right)$ and the corresponding golden rule rates in $\mathbf{R}$ must inevitably fail to reproduce the correct dynamics.

The idea is thus to start from the pseudo-angular momentum basis (14) and to employ separate rate descriptions for each invariant subspace with initial and equilibrium populations according to Eqs. (19) and (29), respectively, and with transition rates chosen according to known expressions like golden rule formulae [18, 19]. The LMBS populations can then be obtained from Eq. (18). This approach, however, only applies for LMBS populations for which the cross terms in Eq. (18) vanish, i.e. if the initial density matrix $\rho(0)$ includes no finite offdiagonal elements with respect to the basis $B_{J}$. In terms of the path-integral picture, this restricts a rate approach to populations whose path-integral expression (37) collapses to a single $A_{J_{1}, J_{1}}(t)$. For instance, for a system obeying Eq. (24) and being prepared according to $P_{-S,-S}(0)=1$ or $P_{S, S}(0)=1$, this holds for all LMBS populations, while for $P_{0,0}(0)=1$ only some of them can be reproduced this way (cf. Figs. 3 and 4). Note that Eq. (24) is no necessary condition for this approach, as we could confirm for a system with $d=3, \epsilon_{i}=0$, $\Delta_{1}=\Delta_{2}, U=5 \Delta_{1}$, and $P_{-1,-1}(0)=1$, where despite a coupling between $\mathcal{G}_{0}$ and $\mathcal{G}_{1}$ all LMBS populations with $s^{\downarrow}+s^{\uparrow} \neq 0$ could be reproduced (not shown).

If, tough, off-diagonal terms are present in $\rho(0)$, they by no means can be captured by a simple rate formalism, and the dynamics of the corresponding LMBS populations will, at least for short to intermediate times, escape a description along the lines of Eq. (39). This reflects the fact that, although unable to introduce transitions between them, the phonon-induced coupling still correlates the dynamics on otherwise disjunctive subspaces, an effect clearly beyond the scope of rate models. To what extent the presence of non-diagonal elements of the density matrix do influence the dynamics in specific situations, however, necessitates a deeper analysis based on the exact path integral expression (34) and a generalization of what is known about standard spin-boson type of models [7]. Nevertheless, we heuristically found that in the cases we investigated, LMBS populations which cannot be solely expressed in terms of pseudo-angular momentum populations still can be well approximated by

$$
\tilde{P}_{s^{\downarrow}, s^{\uparrow}}(t) \equiv \bar{P}_{s^{\downarrow}, s^{\uparrow}}(t)+\left(1-e^{-\gamma t}\right)\left(P_{s^{\downarrow}, s^{\uparrow}}^{\infty}-\bar{P}_{s^{\downarrow}, s^{\uparrow}}^{\infty}\right),
$$

where $\bar{P}_{s^{\downarrow}, s^{\uparrow}}(t)$ denotes the corresponding LMBS population as obtained from Eq. (39), and $\gamma$ is the small- 
est finite modulus of the eigenvalues of the rate matrices of all invariant subspaces $\mathcal{G}_{j}$ which participate in the dynamics (cf. Fig. 4). Whether Eq. (40) also covers a more general set of parameters, however, lays beyond our knowledge as well as an explanation for observation that in cases where no finite non-diagonal elements are present, Eq. (40) performs rather poorly. Thus, Monte Carlo simulations are of particular importance to obtain insight into the nonequilibrium dynamics of correlated charge transfer in cases where symmetries are broken.

\section{APPLICATIONS}

In this section we apply the general results of the previous sections to specific models, particularly, to tightbinding systems with $d=3$ and $d=4$ sites. The simplest case $d=2$ is not explicitly addressed since it has been extensively studied in Ref. 11]. We only mention again that for this generalization of the ordinary spinboson model, a decomposition in $\mathcal{G}^{+} \equiv \mathcal{G}_{1}$ and $\mathcal{G}^{-} \equiv \mathcal{G}_{0}$ exists for arbitrary system parameters, where the singlet state in the latter subspace constitutes a DFS.

\section{A. The case $d=3$}

As already stated above, for isotropic couplings and all on-site energies and the Coulomb energy set to zero, one has a full decomposition of the Hilbert space. Corresponding data for the dynamics of the localized populations (7) are depicted in Figs. 3] and 4 for an ohmic spectral density with exponential cutoff,

$$
J(\omega)=2 \pi \alpha \omega \mathrm{e}^{-\omega / \omega_{c}}
$$

where $\alpha=0.25, \omega_{c}=5 \Delta$, and $\hbar \beta=0.1 \hbar \Delta$ (the bath setup is the same for all populations shown here). Note the change in the equilibrium populations due to the different initial preparations. Turning on the Coulomb interaction, i.e. $U \neq 0$, a coupling between $\left|\psi_{0}^{(0)}\right\rangle$ and $\left|\psi_{0}^{(2)}\right\rangle$ is introduced (cf. Fig. 2), with $\left\langle\psi_{0}^{(2)}\left|H_{S}\right| \psi_{0}^{(0)}\right\rangle=$ $(\sqrt{2} / 3) U$, such that only two invariant subspaces remain, i.e.

$$
\mathcal{H}_{d=3}=\mathcal{G}_{0 \cup 2} \cup \mathcal{G}_{1}
$$

with $\mathcal{G}_{0 \cup 2}=\mathcal{G}_{0} \cup \mathcal{G}_{2}$. However, when fixing the interaction strength to the specific value $U_{C}=\epsilon_{-1}+\epsilon_{1}-2 \epsilon_{0}$, a full decomposition is recovered, see Fig. 5

Note that, without an external magnetic field, even for arbitrary system parameters the particle-exchange symmetry cannot be broken: Albeit $\left|\psi_{0}^{(0)}\right\rangle$ then typically couples to $\left|\psi_{-}^{(2)} 1\right\rangle,\left|\psi_{0}^{(2)}\right\rangle$, and $\left|\psi_{1}^{(2)}\right\rangle$, no couplings between angular-momentum states with even and odd $J$ exist, such that always a non-Boltzmann distribution for the equilibrium populations is obtained (cf. Fig. 6).

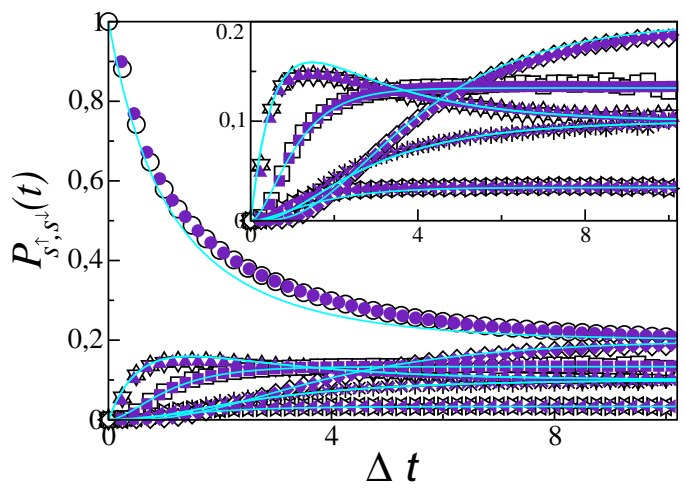

FIG. 3: Initial dynamics of a two-particle system for $d=$ 3 with completely degenerate onsite energies and coupling strengths $\Delta_{1}=\Delta_{2}=\Delta$ and $U_{C}=0$. Shown are PIMC data for the localized populations Eq. (7) as obtained from Eq. (34) (empty black symbols) and Eq. (37) (filled gray symbols); gray lines denote results from the rate approach described in Sec. VIB Shown are $P_{-1,-1}$ (circles), $P_{-1,0}$ (triangles up), $P_{-1,1}$ (triangles left), $P_{0,-1}$ (triangles down), $P_{0,0}$ (squares), $P_{0,1}$, ("+"), $P_{1,-1}$ (triangles right), $P_{1,0}$ ("×"), and $P_{1,1}$ (diamonds), with equilibrium values of $1 / 5,1 / 10,1 / 30,1 / 10$, $2 / 15,1 / 10,1 / 30,1 / 10$, and $1 / 5$, respectively. The inset exhibits are more detailed view of the populations with no initial population.

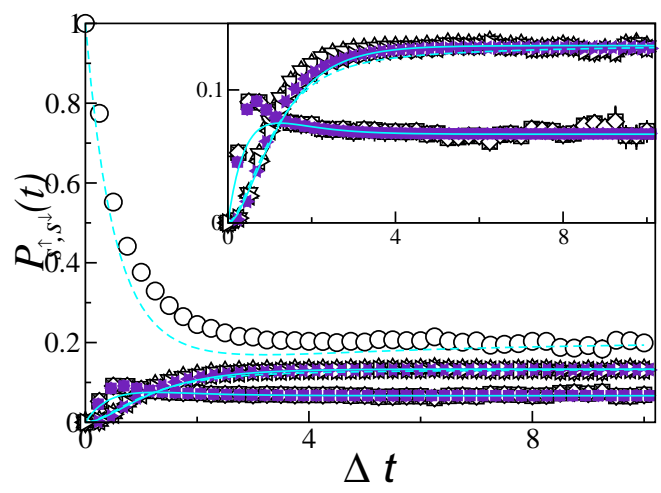

FIG. 4: Same as Fig. 3 but with initial preparation on $|0,0\rangle$. Shown are $P_{-1,-1}$ (triangles left), $P_{-1,0}$ (squares), $P_{-1,1}$ (triangles up), $P_{0,-1}$ ("+"), $P_{0,0}$ (circles), $P_{0,1}$, ("×"), $P_{1,-1}$ (triangles down), $P_{1,0}$ (diamonds), and $P_{1,1}$ (triangles right), with equilibrium values of $2 / 15,1 / 15,2 / 15,1 / 15,1 / 5,1 / 15$, $2 / 15,1 / 15$, and $2 / 15$, respectively. Dashed gray lines denote $\tilde{P}_{-1,1}(t), \tilde{P}_{0,0}(t)$, and $\tilde{P}_{1,-1}(t)$ (40).

\section{B. The case $d=4$ : Example for a partial decomposition}

For a tight-binding lattice with $d=4$ sites, a full decomposition of the Hilbert space is already not possible for vanishing energies and isotropic hopping terms. Nev- 


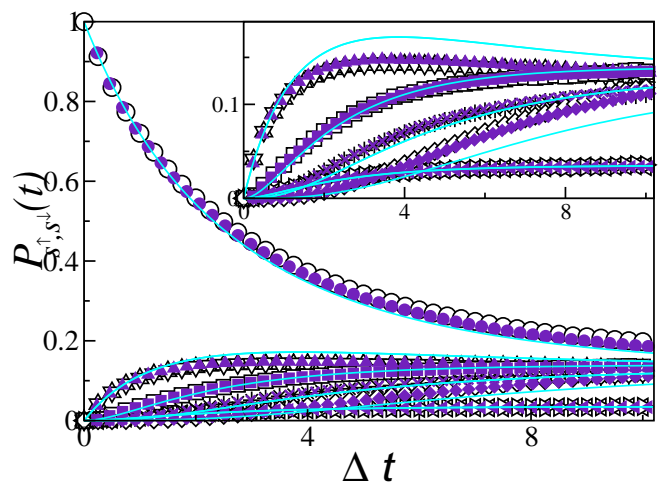

FIG. 5: Same as Fig. 3 but with $\epsilon_{1}=\epsilon_{3}=0, \epsilon_{2}=-2.5 \Delta$, and $U_{C}=5 \Delta$, with equilibrium values of $0.1269,0.1343$, $0.0349,0.1343,0.1394,0.1343,0.0349,0.1343$, and 0.1269 .

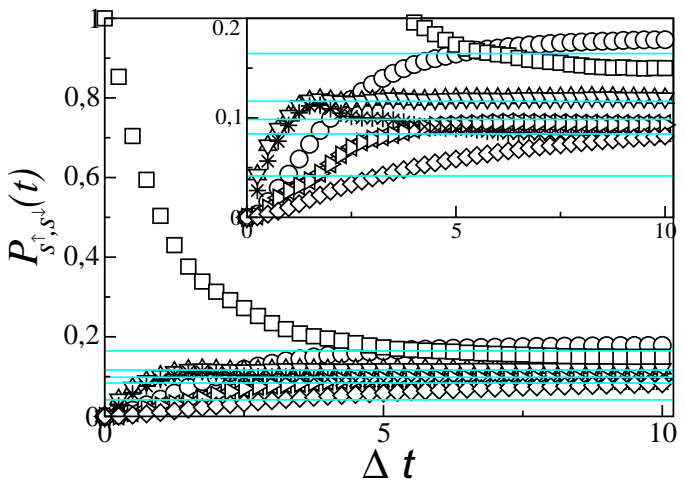

FIG. 6: Same as Fig. 3] but with $\Delta_{2}=0.7 \Delta_{1}, \epsilon_{1}=-1.65 \Delta_{1}$, $\epsilon_{2}=0, \epsilon_{3}=3.5 \Delta_{1}$, and $U_{C}=5.1$, with equilibrium values of $0.1884,0.1330,0.0812,0.1330,0.1567,0.0795,0.0812,0.0795$, and 0.0673. Gray lines here denote equilibrium populations as obtained from a Boltzmann distribution if all symmetries were broken.

ertheless, one can still find a partial decomposition

$$
\mathcal{H}_{d=4}=\mathcal{G}_{0} \cup \mathcal{G}_{2} \cap \mathcal{G}_{1 \cup 3},
$$

where $\mathcal{G}_{1 \cup 3}=\mathcal{G}_{1} \cup \mathcal{G}_{3}$, and $\mathcal{G}_{0}, \mathcal{G}_{2}$, and $\mathcal{G}_{1 \cup 3}$ are again disjunctive and invariant with respect to $H$ (see Fig. 17). A Boltzmann distribution again can be observed for the equilibrium distribution of $\left|\psi_{m}^{(j)}\right\rangle$ states for each invariant subspace but not for the total Hilbert space. Contrary to $\mathcal{G}_{1}$ and $\mathcal{G}_{3}$, a combination of invariant subspaces like $\mathcal{G}_{1 \cup 3}$ will in general not exhibit a linear structure with respect to the tunnel couplings. For a energetically nondegenerate systems, further couplings between $\mathcal{G}_{0}$ and $\mathcal{G}_{2}$ are introduced. However, even for arbitrary on-site energies $E_{i}$, Coulomb energies $U$, and tunnel couplings $\Delta_{i}$ the decomposition of the Hilbert space can never be completely destroyed due to the underlying spin-permutation symmetry discussed in Sec. III

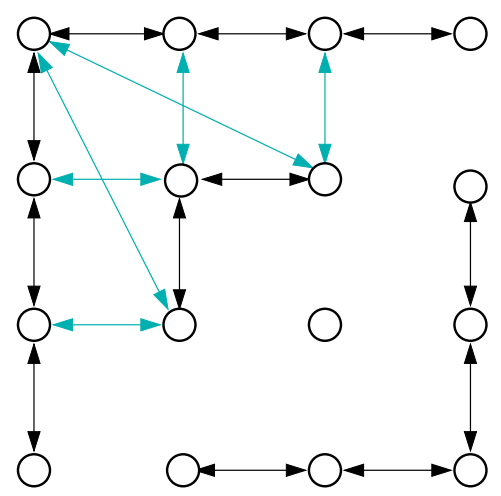

FIG. 7: 2d mapping for an energetically degenerate $d=$ 4 chain. Black solid lines: Couplings for $\Delta_{3}=\Delta_{1}, \Delta_{2}=$ $2 / \sqrt{3} \Delta_{1}$; gray lines: Additional couplings for $\Delta_{3}=\Delta_{2}=\Delta_{1}$.

\section{Quasi-invariant subspaces}

For systems with a high symmetry (e.g. when Eq. (24) applies), even a minute change in the system's parameters can lead to a symmetry breaking. Along goes a modification of the structure of the Hilbert space: Formerly invariant subspaces merge such that their number decreases. Since the size of the new invariant subspaces exceeds that of the former ones, the equilibration process within such a larger subspace is expected to slow down accordingly. If, however, the coupling between formerly invariant subspaces remains rather small, the relaxation process may in fact occur on two (or more) time scales: One (or more) governing the internal dynamics within each former subspace and another one, strongly separated from those, governing the overall approach to equilibrium. In this situation, one may still speak of quasi-invariant subspaces which establish quasistationary states on an intermediate time scale.

For example, a weak particle interaction with $U_{C}=$ $0.05 \Delta$ in an otherwise energetically degenerate system with $d=3$ leads to a weak coupling between $\left|\psi_{0}^{(0)}\right\rangle$ and $\left|\psi_{0}^{(2)}\right\rangle$ (cf. Fig. 2). Consequently, instead of the formerly invariant subspaces $\mathcal{G}_{0}, \mathcal{G}_{1}$, and $\mathcal{G}_{2}$, now only $\mathcal{G}_{1}$ and $\mathcal{G}_{0 \cup 2}=\mathcal{G}_{0} \cup \mathcal{G}_{2}$ remain invariant with respect to $H$. The smallest eigenvalue of the according rate matrix $\mathbf{R}$ (see Sec. (VIB), describing the long-time dynamics 19], then becomes $0.010 \Delta$, suggesting an equilibration timescale of the order of $100 \Delta^{-1}$. The second smallest eigenvalue, however, exceeds the latter by a factor of more than 20. Accordingly, for $1 \ll \Delta t \ll 100$, the system will be trapped in a 'quasi-equilibrium', which still reflects the full symmetry and therefore significantly differs from the true equilibrium state (cf. Fig. 8). From an experimental point of view, this quasi-equilibrium might easily be mistaken as the true equilibrium since on the one hand, the equilibration timescale can take very large values for systems with only slightly broken symmetries, while on the 


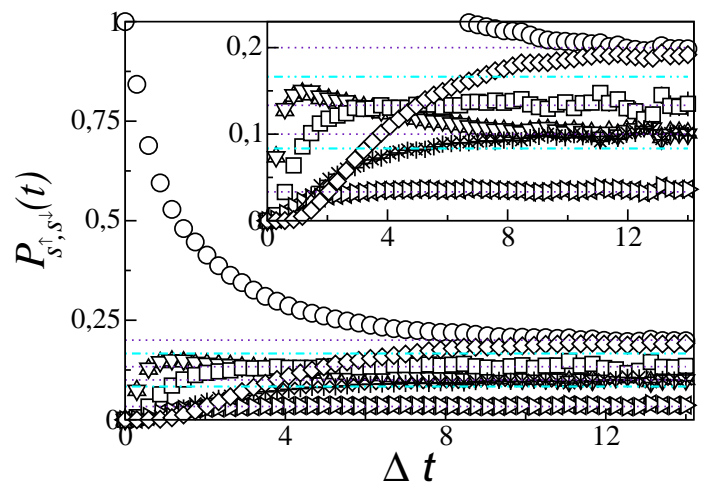

FIG. 8: Same as in Fig. 3 but with $U_{C}=0.05 \Delta$. The gray dot-dashed lines show the equilibrium populations $P_{-1,-1}^{\infty}=$ $P_{1,1}^{\infty}=0.1663$ (circles and diamonds), $P_{-1,0}^{\infty}=P_{0,-1}^{\infty}=$ $P_{0,1}^{\infty}=P_{1,0}^{\infty}=0.0835$ (triangles up, triangles down, "+", and " $\times$ "), 0.0834 (triangles left and triangles right), and 0.1666 (squares), respectively. Solid dark-gray dotted lines represent the equilibrium populations for a corresponding system with $U_{C}=0$.

other such a symmetry breaking is rather hard to identify since it can easily occur at rather 'harmless' system parameters. Note that this phenomenon can also occur when applying a weak magnetic field which breaks the spin-permutation symmetry.

\section{Particle interaction}

An unique feature of many-particle systems is the interaction between its constituents, where the Coulomb interaction is the most prominent example. In our model (11)-(3), Coulomb interaction between particles manifests itself as change of the energies of the localized states and influences the dissipative system in quite different ways: On the one hand, it can facilitate or break the symmetries discussed in Secs. III and IV and accordingly the decomposition of the full Hilbert space into invariant subspaces, as has been intensively discussed above. On the other, as has been already pointed out by R. Marcus [28], a different energy offset between adjacent sites $\Delta E_{j}=E_{j}-E_{j+1}$ introduces a phonon activation barrier between these sites, which is a unique signature of the interaction between system and phonon bath. It reflects the fact that for a transition between adjacent sites energy fluctuations are necessary for the reorganization of the bath degrees of freedom after the tunneling of an electron. This latter process can exhibit a strong impact, since for larger Coulomb interaction $U_{j}$, the phonon activation barrier exceeds the electronic offset $\Delta E_{j}$ by far. In fact, it can lead to rather counter-intuitive dynamics: If, for example, two electrons are initially prepared on the same site of the chain, any Coulomb interaction is expected to speed up the initial state's depopulation.

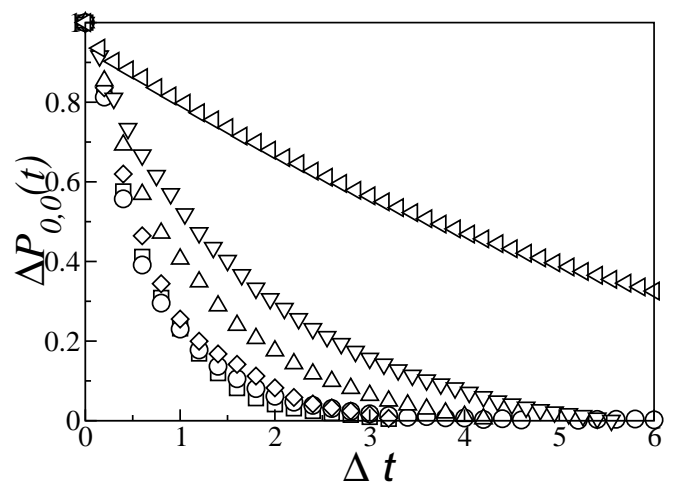

FIG. 9: $\Delta P_{0,0}(t)$ for a system with $d=3, \epsilon_{i}=0$, and $U_{C} / \Delta=0$ (circles), 2.5 (squares), 5 (diamonds), 7.5 (triangles up), 10 (triangles down), 15 (triangles left), and 20 (triangles right), initially prepared in $|0,0\rangle$.

However, in competition to that the phonon interaction also generates an activation barrier, which in turn slows down any transitions between the initial and neighboring states. Therefore, only sufficiently weak Coulomb repulsions will indeed lead to a faster emptying of the initial state; large repulsion strengths, in contrast, cause a slow-down of the initial depopulation dynamics and can even lead to a stabilization of the initial state. Figure 9 visualizes this by comparing the initial dynamics of systems with varying interaction strengths $U_{C}$. Note that the turnaround takes places at around $U_{C}=2.5 \hbar \Delta$, which coincides with the classical reorganization energy $\hbar \Lambda^{(c l)}=(1 / \pi) \int_{0}^{\infty} d \omega J(\omega) / \omega$.

We note in passing that a quantitative discussion of the effect of phonon-induced interactions via the common environment is, unlike to the case of two indistinguishable particles, not easy: While in the latter case one could simply compare the dynamics of two particles on a $1 \mathrm{~d}$ chain with Coulomb repulsion $U_{C}$ with the QMC data for the dynamics of a single particle on a corresponding $2 \mathrm{~d}$ lattice [26], for two distinguishable particles this approach is of no avail due to the intrinsically completely different structures of the Hilbert spaces.

\section{CONCLUSIONS}

In this paper we have analysed the correlated nonequilibrium dynamics of two interacting distinguishable fermions on a one-dimensional tight binding lattice embedded into a phononic environment. As a major result, we have revealed the crucial role of symmetries and provided their full characterization. In particular, there is: (i) a spin-permutation symmetry which is fundamental in that it is conserved independent of system and bath parameters; it leads to a separation of the full Hilbert space into two disjunct subspaces. (ii) A conservation of a total quasi-angular momentum $\vec{J}^{2}$ corresponding to 
$\left[H, \vec{J}^{2}\right]=0$ for certain topologies of system parameters. This symmetry determines a set of $J=d$ ( $d$ number of sites) disjunct subspaces. The dissipative many-body dynamics happens to occur in each of these subspaces independently with transitions between them only mediated by the initial preparation and/or the measurement observable. Recently, similar kinds of symmetries have been discussed for quantum state transfer along networks as realized e.g. in linear arrays of Josephson junctions 29]. In any case, the time evolution in presence of the heat bath is not ergodic moving towards a stationary state which is not Boltzmann like distributed. Of particular relevance is the DFS, a subspace with no coupling to the bath at all, the initial state of which is preserved for all times. For $d=2$ the nature of the many body DFS is equivalent to the DFS discussed in the context of quantum information processing for interacting spin- $1 / 2$ systems, but differs from the latter for all $d>2$.

A direct practical consequence of the symmetry properties is that the dynamical sign problem notoriously occurring in real-time quantum Monte Carlo approaches can be substantially damped by performing the sampling in each of the subspaces separately. Further, a rate description developed for single particle tight binding systems and known to be applicable whenever the dynamics is sufficiently incoherent, could be generalized to capture also the many body relaxation. This approximation is of great importance as it not only allows to obtain a deeper physical insight into the numerical QMC data, but also to investigate the long-time domain which is typically not accessible by the PIMC.

We have illustrated these findings for one-dimensional Hubbard chains with sites of up to $d=4$ and considerably long times, which may be also of experimental interest. In particular, small arrays of quantum dots would be appropriate candidates, where the above symmetries could directly be exploited to control dynamical features of two electron transport. In fact, a fully controllable device consisting of two coupled quantum dots has already been realized [2]. An additional external magnetic field further opens a way to break or restore the fundamental spin-permuation symmetry. Note that although here we have been mostly concerned with incoherent dynamics, the above findings also apply to the domain of coherent transport as shown for $d=3$ in Ref. [11].

Systems with more than two electrons or systems with indistinguishable particles can be analysed along the same lines as above, however, with differences in detail. In this context the case of many boson systems coupled to a dissipative bath, i.e. a bosonic Hubbard model with dissipation, is certainly of great interest, e.g. for condensed ultra-cold atomic gases. Recently, we have already shown that for a two boson system a DFS exists only for an odd number of lattice sites [26]. Work to extend this study to a full characterization of the symmetry properties as well as a generalization of the rate description are currently in progress.

\section{Acknowledgments}

We thank A. Komnik and H. Grabert for fruitful discussions. This work has been supported by grants from the Ministry of Science, Research and Arts of Baden Württemberg No. 24-7532.23-11-11/1 and from the DFG. JA acknowledges a Heisenberg fellowship of the DFG.

\section{APPENDIX A: DISCRETIZED PATH INTEGRAL FOR MANY-BODY SYSTEMS}

The derivation of a discretized version of the pathintegral expression (34) is very similar to the corresponding case of a single particle (see, e.g., Refs [7, 17, 18, 19]). Therefore, we here restrict ourselves mainly to details specific to the many-particle case.

We begin with inserting $2 q$ complete sets of projectors, one after each short-time step $\tau \equiv t / q$,

$$
\begin{aligned}
A(t)= & \operatorname{tr}\{W(t) A\} \\
= & \sum_{\left|s_{j}^{\downarrow}\right|,\left|s_{j}^{\uparrow}\right| \leq S}\left(\prod_{j=2}^{2 q} \int d \vec{x}_{j}\right)\left\langle\vec{s}_{1}, \vec{x}_{1}|W(0)| \vec{s}_{2}, \vec{x}_{2}\right\rangle \\
& \times \prod_{j=3}^{q}\left\langle\vec{s}_{j-1}, \vec{x}_{j-1}\left|e^{i \tau H / \hbar}\right| \vec{s}_{j}, \vec{x}_{j}\right\rangle \\
& \times\left\langle\vec{s}_{q}, \vec{x}_{q}|A| \vec{s}_{q+1}, \vec{x}_{q+1}\right\rangle \\
& \times \prod_{j=q+2}^{2 q}\left\langle\vec{s}_{j-1}, \vec{x}_{j-1}\left|e^{-i \tau H / \hbar}\right| \vec{s}_{j}, \vec{x}_{j}\right\rangle,
\end{aligned}
$$

where $|\vec{x}\rangle$ is a suitable basis of the environment, and $\vec{s} \equiv$ $\left(s^{\downarrow}, s^{\uparrow}\right)$ denotes the system's coordinates with respect to $\mathcal{B}_{\text {loc }}$ (5). Exploiting the symmetric Suzuki splitting,

$$
\begin{aligned}
\left\langle\vec{s}_{j-1},\right. & \left.\vec{x}_{j-1}\left|e^{i \tau H / \hbar}\right| \vec{s}_{j}, \vec{x}_{j}\right\rangle \\
= & \left\langle\vec{s}_{j-1}, \vec{x}_{j-1}\right| e^{i \tau\left(H_{I}+H_{B}\right) / 2 \hbar} \\
& \times e^{i \tau H_{S} / \hbar} e^{i \tau\left(H_{I}+H_{B}\right) / 2 \hbar}\left|\vec{s}_{j}, \vec{x}_{j}\right\rangle+O\left(\tau^{3}\right) \\
= & \exp \left\{\frac{i a^{2} \tau}{\hbar}\left[\left(\vec{s}_{j-1} \cdot \vec{e}\right)^{2}+\left(\vec{s}_{j-1} \cdot \vec{e}\right)^{2}\right] \sum_{\alpha} \frac{c_{\alpha}^{2}}{2 m_{\alpha} \omega_{\alpha}^{2}}\right\} \\
& \times K\left(\vec{s}_{j-1}, \vec{s}_{j}\right) \\
\quad & \left\langle\vec{x}_{j-1}\right| \exp \left\{\frac{i \tau}{2 \hbar}\left[-a\left(\vec{s}_{j-1} \cdot \vec{e}\right) \sum_{\alpha} c_{\alpha} X_{\alpha}+H_{B}\right]\right\} \\
& \quad \times \exp \left\{\frac{i \tau}{2 \hbar}\left[-a\left(\vec{s}_{j} \cdot \vec{e}\right) \sum_{\alpha} c_{\alpha} X_{\alpha}+H_{B}\right]\right\}\left|\vec{x}_{j}\right\rangle \\
+ & O\left(\tau^{3}\right),
\end{aligned}
$$

where

$$
K\left(\vec{s}_{j-1}, \vec{s}_{j}\right) \equiv\left\langle\vec{s}_{j-1}\left|e^{i \tau H_{S} / \hbar}\right| \vec{s}_{j}\right\rangle
$$

denotes the free system's propagator over a time interval $\tau$, one obtains an expression where the environmental 
degrees of freedom can be integrated out exactly due to the harmonic nature of $H_{B}$ and $H_{I}$, leading to a discretized version $\Phi\left[\left\{\vec{s}_{j}\right\}\right]$ of the influence functional (35). Since, after replacing $\vec{s}_{j} \cdot \vec{e}$ with the corresponding singleparticle coordinate, the environmental terms in Eq. (A2) are then identical to those of the single-particle case (cf., e.g., Ref [17, 18, 19]), $\Phi\left[\left\{\vec{s}_{j}\right\}\right]$ can be easily obtained from the single-particle expression without the need of redoing the tedious integrals. Rewriting the system's coordinates with the aid of the forward and backward paths introduced in Sec. VIA

$$
\begin{aligned}
& s_{j}^{\downarrow / \uparrow} \rightarrow s_{j}^{\downarrow / \uparrow} \quad \text { for } 1 \leq j \leq q+1, \\
& s_{j}^{\downarrow / \uparrow} \rightarrow \tilde{s}_{2 q+2-j}^{\downarrow / \uparrow} \text { for } q+2 \leq j \leq 2 q+1,
\end{aligned}
$$

the exponent of the influence functional becomes, in terms of the sum and difference coordinates $\vec{\eta}_{j} \equiv\left(s_{j}^{\downarrow}+\right.$ $\left.\tilde{s}_{j}^{\downarrow}, s_{j}^{\uparrow}+\tilde{s}_{j}^{\uparrow}\right)$ and $\vec{\xi}_{j} \equiv\left(s_{j}^{\downarrow}-\tilde{s}_{j}^{\downarrow}, s_{j}^{\uparrow}-\tilde{s}_{j}^{\uparrow}\right)$, respectively,

$$
\begin{aligned}
\Phi\left[\left\{\vec{\eta}_{j}, \vec{\xi}_{j}\right\}\right]= & i(\eta(0) \cdot \vec{e}) \sum_{j=2}^{q}\left(\vec{\xi}_{j} \cdot \vec{e}\right) \hat{X}_{j} \\
& +\sum_{j \geq k=2}^{q}\left\{\left(\vec{\xi}_{j} \cdot \vec{e}\right) \Lambda_{j-k}\left(\vec{\xi}_{k} \cdot \vec{e}\right)\right. \\
& \left.+i\left(\vec{\xi}_{j} \cdot \vec{e}\right) X_{j-k}\left(\vec{\eta}_{k} \cdot \vec{e}\right)\right\} .
\end{aligned}
$$

The real valued $\ldots \Lambda_{j}, X_{j}$, and $\hat{X}_{j}$ are calculated according to

$$
\begin{aligned}
\Lambda_{0}+i X_{0}= & Q(\tau), \\
\Lambda_{l}+i X_{l}= & Q((l-1) \tau)+Q((l+1) \tau)-2 Q(l \tau) \\
& \text { for } 1 \leq l \leq q-2, \\
\hat{X}_{j}= & \operatorname{Im}\{Q((j-2) \tau)-Q((j-1) \tau)\} \\
& \text { for } 2 \leq j \leq q,
\end{aligned}
$$

where

$$
\begin{gathered}
Q(t)=\frac{1}{\pi} \int_{0}^{\infty} d \omega \frac{J(\omega)}{\omega^{2}}\{\operatorname{coth}(\hbar \beta \omega / 2)[1-\cos (\omega t)] \\
+i \sin (\omega t)\}
\end{gathered}
$$

is the twice integrated bath-autocorrelation function $L(t)$ 7]. We thus arrive, as a discretized version of Eq. (34), at

$$
\begin{gathered}
\langle A(t)\rangle=\sum_{\left\{\vec{\eta}_{j}, \vec{\xi}_{j}\right\}} a\left(\vec{\eta}_{q+1}\right) \prod_{j=2}^{q} K\left(\vec{\eta}_{j}, \vec{\xi}_{j} ; \vec{\eta}_{j+1}, \vec{\xi}_{j+1}\right) \\
\quad \times \exp \left\{-\Phi\left[\left\{\vec{\eta}_{j}, \vec{\xi}_{j}\right\}\right]\right\},
\end{gathered}
$$

which can now readily evaluated with PIMC schemes.

Caldas, M. B. Nardelli, and E. Molinari, Phys. Rev. Lett. 94, 116802 (2005).

[15] E. G. Petrov and P. Hänggi, Phys. Rev. Lett. 86, 2862 (2001).

[16] F. Kaiser, M. Strass, S. Kohler, and P. Hänggi, Chem. Phys. 322, 193 (2006).

[17] R. Egger and C. Mak, Phys. Rev. B 50, 15210 (1994).

[18] L. Mühlbacher, J. Ankerhold, and C. Escher, J. Chem. Phys. 121, 12696 (2004).

[19] L. Mühlbacher and J. Ankerhold, J. Chem. Phys. 122, 184715 (2005).

[20] M. Suzuki, Quantum Monte Carlo Methods in Condensed Matter Physics (World Scientific, 1993).

[21] J. Hirsch and D. Scalapino, Phys. Rev. B 29, 5554 (1984).

[22] J. Schulte and M. Böhm, Phys. Rev. B 53, 15385 (1996).

[23] J. Hirsch, Phys. Rev. B 65, 214510 (2002).

[24] S. Tornow, N.-H. Tong, and R. Bulla, cond-mat/0502276 (2005).

[25] A. O. Caldeira and A. J. Leggett, Physica A 121, 587 (1983).

[26] L. Mühlbacher, C. Escher, and J. Ankerhold, Chem. Phys. 322, 200 (2006).

[27] J. Sakurai, Modern Quantum Mechanics (AddisonWesley, 1994).

[28] R. A. Marcus, J. Chem. Phys. 24, 966 (1956).

[29] S. Bose, Phys. Rev. Lett. 91, 207901 (2003).

[13] Y. Meir and N. Wingreen, Phys. Rev. Lett. 68, 2512 (1992).

[14] A. Ferretti, A. Calzolari, R. D. Felice, F. Manghi, M. J. 